\title{
Ausschreibungen 2022
}

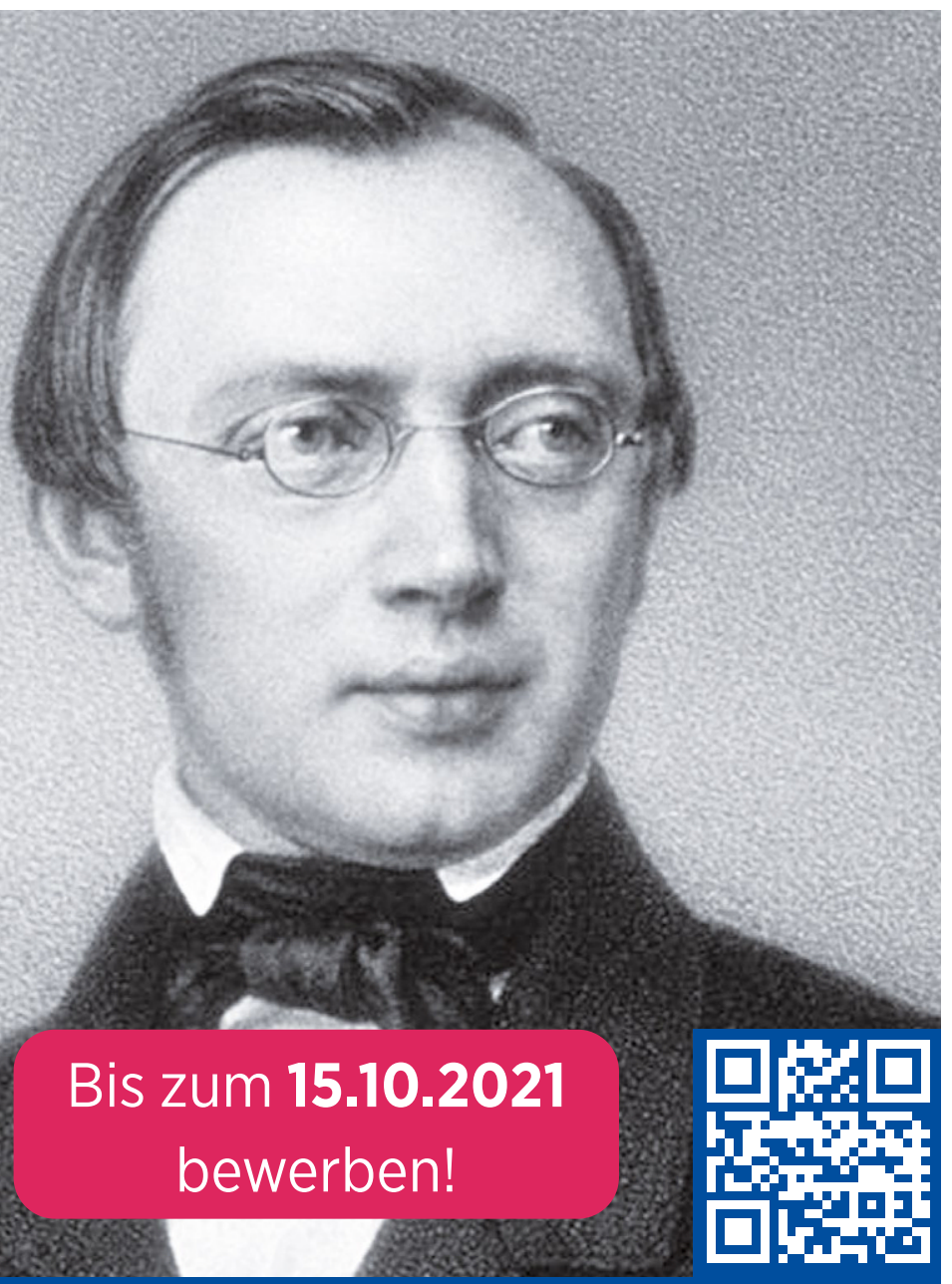

Theodor-FrerichsPreis

» Für die beste vorgelegte, möglichst klinisch - experimentelle Arbeit auf dem Gebiet der Inneren Medizin

» Dotiert mit 30.000€

» Alle Informationen unter www.dgim.de/frerichspreis

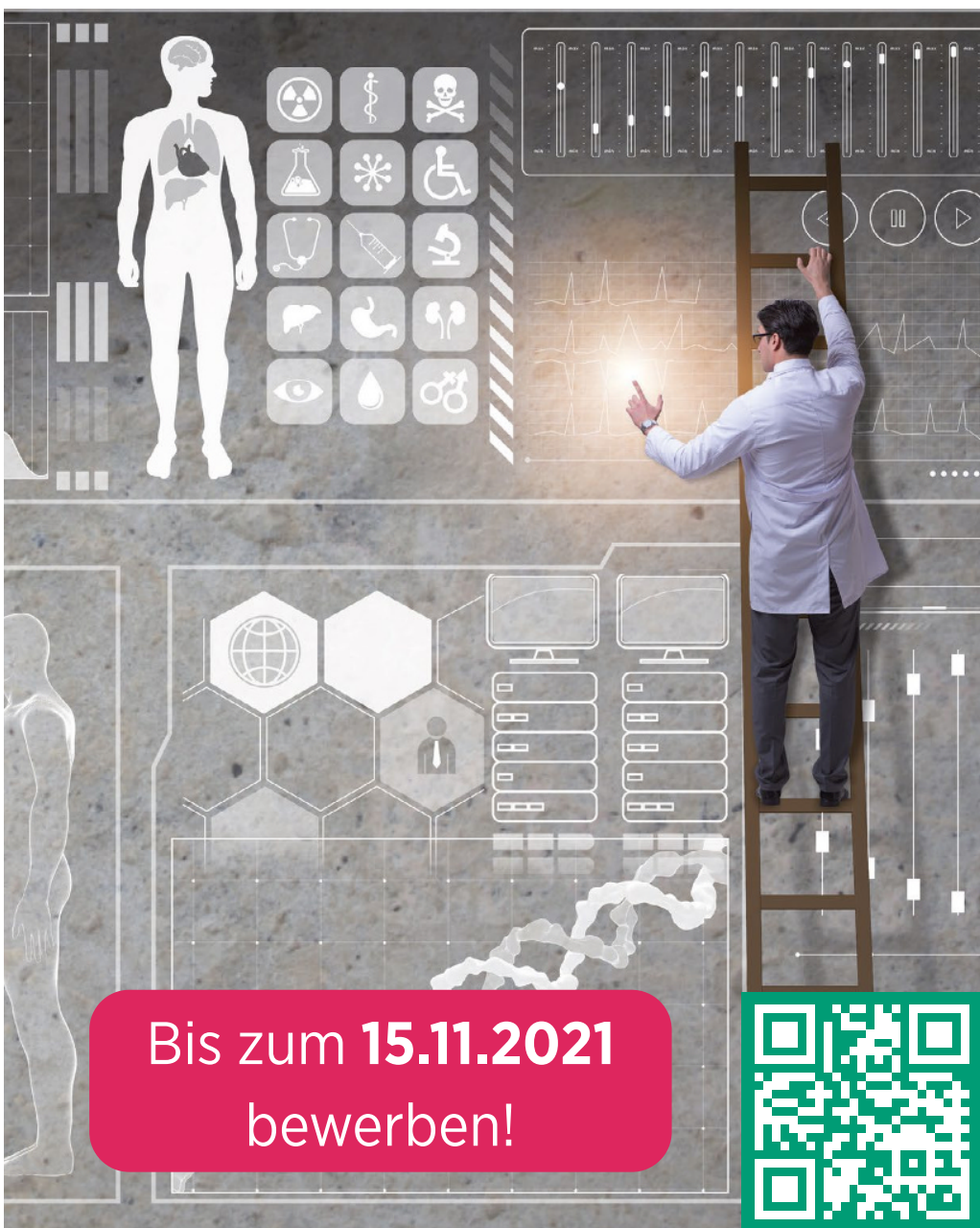

\section{Präventions- Preis}

» Für die beste Arbeit auf dem Gebiet der Primär- und Sekundärprävention innerer Erkrankungen, aus allen Ebenen der klinischen Forschung.

» Dotiert mit 10.000€

» Alle Informationen unter www.dgim.de/stiftung 
Inhalt

$\begin{array}{ll}\mathbf{9 6 9} & \text { Versorgungslandschaft Innere Medizin in Deutschland } \\ \mathbf{9 7 3} & \text { Arbeitsgemeinschaft Transition - ein Update } \\ \mathbf{9 7 3} & \text { DGIMTalk am 06.09.2021: Lehren aus Corona } \\ \mathbf{9 7 5} & \text { 65 Freikarten für Update-Seminare } \\ \mathbf{9 7 5} & \text { DGIM hat nun 28.000 Mitglieder } \\ \mathbf{9 7 5} & \text { DGIM Ausschreibungen } 2022 \\ \mathbf{9 7 6} & \text { 58. Kongress Südwestdeutsche Gesellschaft für Innere Medizin }\end{array}$

Projekt der DGIM-Kommission „Struktur Krankenversorgung"

\section{Versorgungslandschaft Innere Medizin in Deutschland}

Die Analyse der Versorgungssituation in der Inneren Medizin und ihrer Schwerpunkte ist die Grundlage zur weiteren Entwicklung von datenbasierten Strategien, die dann in den politischen Diskurs eingebracht werden können. Ziel der Kommission "Struktur Krankenversorgung" der DGIM ist daher, einen Datensatz zu erheben und ggf. regelmäßig zu aktualisieren, der eine bedarfsorientierte Planung von Versorgungsstrukturen in der Inneren Medizin ermöglicht.

Gesundheitspolitische Steuerung erfolgt in Deutschland im Wesentlichen föderal, teils im Rahmen der Selbstverwaltung, teils durch Krankenhausplanung und DRG-System im stationären Bereich sowie Bedarfsplanung und EBM-System im ambulanten Bereich. Dabei orientiert sich die Steuerung überwiegend am Ressourcenverbrauch und nicht primär medizinisch bzw. bedarfsorientiert. Demzufolge richtet sich auch die Entwicklung der Schwerpunkte der Inneren Medizin häufig nach ökonomischen Kriterien und nicht primär nach medizinischer Qualität und Patientenbedarf. Dies birgt Potenzial für kurz- und längerfristige Fehlentwicklungen im Gesundheitswesen mit indirekten Implikationen auch für den Nachwuchs, die Weiterbildung und nicht zuletzt die Forschung.

Die Kommission „Struktur Krankenversorgung“ wurde von der DGIM mit einem Projekt beauftragt, dessen Konzept im Folgenden kurz vorgestellt werden soll. Es soll in Zusammenarbeit mit dem Fachgebiet "Management im Gesundheitswesen“ der Technischen Universität Berlin, für die Innere Medizin und ihre Schwerpunkte dargestellt werden, wie die Fachdisziplinen innerhalb der Strukturen des Gesundheitssystems ausgestattet sind und welches Leistungsspektrum sie im ambulanten und stationären Sektor abdecken. Mit Hilfe einer soliden Datengrundlage können wir ein um-

Internist 2021 · 62:968-979

https://doi.org/10.1007/s00108-021-01129-3

(c) Springer Medizin Verlag GmbH, ein Teil von Springer Nature 2021

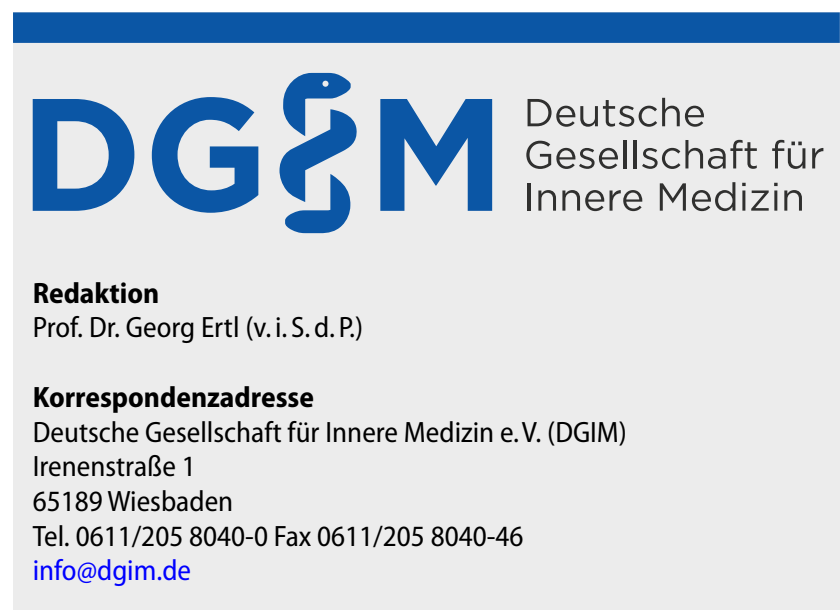

fassendes Bild über die Versorgungssituation in der Fläche gewinnen, um in Folge Über- und Unterversorgung im Verhältnis zum entsprechenden medizinischen „Bedarf“ zu erkennen. Daraus werden sich sicherlich Forderungen für die sachgerechte medizinische Versorgung ergeben.

\section{Strategie des Vorgehens}

Die Darstellung der Versorgungslandschaft der Inneren Medizin in Deutschland soll in einem mehrstufigen Prozess und anhand einer Analyse

- der Ärztinnen und Ärzte,

- der Strukturen, in denen sie tätig sind,

- und der behandelten Patienten

in allen Schwerpunkten der Inneren Medizin für den stationären und ambulanten Bereich zunächst auf bundesweiter Ebene (ggf. später auch auf Länderebene) erfolgen (• Abb. 1). Zudem werden die Schnittstellen zwischen ambulant und stationär hinsichtlich ihrer personellen Strukturen und des Fallaufkommens betrachtet. Abschließend und zur Ermittlung eines möglichen Umstrukturierungspotentials ist ein internationaler Vergleich vorgesehen.

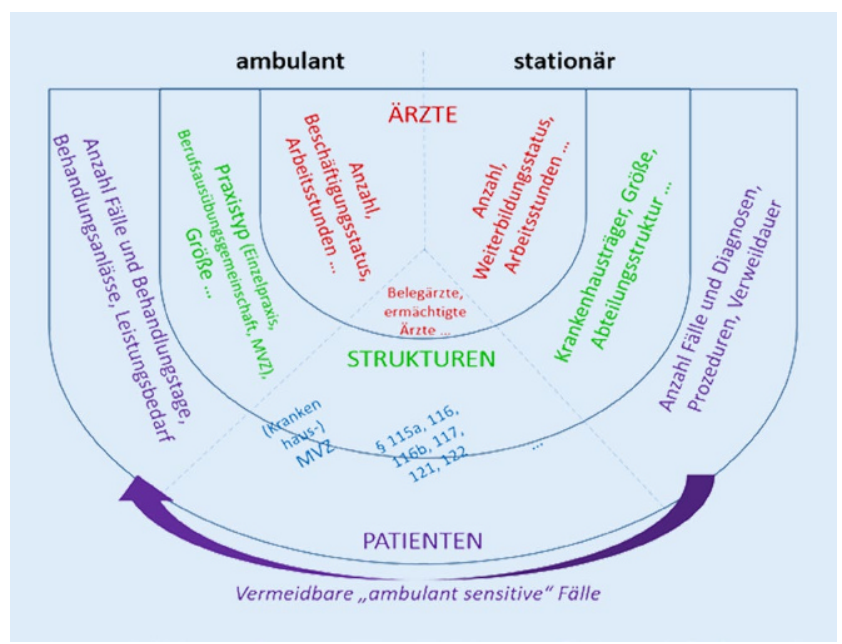

Abb. $1 \Delta$ Konzept und Ebenen der Analysen des Projekts 


\section{Ärztinnen \& Ärzte der IM nach Tätigkeitsbereichen}

- von rund 410.000 ärztlich tätigen Ärztinnen/Ärzten in 2020 gehören gut $\mathbf{5 8 . 0 0 0 ~ d e r ~ I M ~ a n ~}(=\mathbf{1 4} \%)$

(o. Gebietsbezeichnung: $30 \%$; Allgemeinmedizin: $11 \%$ )

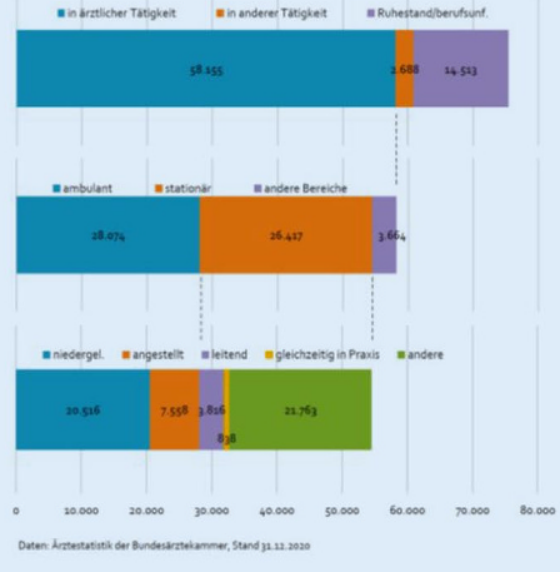

Abb. $2 \triangleleft$ Tätigkeitsbereiche der Ärztinnen und Ärzte im Jahr 2020 IM Innere Medizin

\section{Methodik des Vorgehens: Analyse des Ist-Standes und der Entwicklung}

Soweit dies mit der gegebenen Datenlage möglich und zielführend ist, erfolgen die Analysen sowohl hinsichtlich des Status quo als auch hinsichtlich ihrer zeitlichen Entwicklung (Verlauf über 5 bis 10 Jahre). Die Quellen der Daten sind im Infokasten zusammengefasst. Als ein erstes Beispiel sind die Tätigkeitsbereiche der Ärztinnen und Ärzte in der Inneren Medizin in - Abb. 2 für das Jahr 2020 graphisch zusammengefasst. In Abhängigkeit von der Datenlage wird ergänzend die Anzahl der sich in Weiterbildung befindlichen Ärzte und Ärztinnen aktuell und in ihrer zeitlichen Entwicklung untersucht.
Für die Darstellung der Situation der Inneren Medizin im stationären Sektor werden Daten aus der Datenbank des Projektes Qualitätstransparenz herangezogen. Sie enthält auf Abteilungsebene u. a. Angaben zur Anzahl an angestellten Ärzten und Fachärzten sowie den Fallzahlen (DRGs und/oder OPS). Die Datenbank basiert auf den Daten der strukturierten Qualitätsberichte und umfasst alle deutschen Krankenhäuser seit 2006. Dafür sollen die häufigsten DRGs/OPS in den jeweiligen Schwerpunkten im Zeitverlauf analysiert werden. Darüber hinaus wird soweit möglich auch erhoben, wie die Leitung von Fachabteilungen ausgestaltet ist.

Für die Analyse des ambulanten Sektors werden Daten der Kassenärztlichen Bundesvereinigung, des Zentralinstituts der kassenärztlichen Versorgung und der Bundesärztekammer verwendet.

\begin{tabular}{|c|c|}
\hline Quelle & Inhalt/Verwendung \\
\hline Ärztestatistik BÄK & - Tätigkeitsbereich von Ärzten nach Facharztgruppe, inkl. SP ab 1996 \\
\hline $\begin{array}{l}\text { ASV-Bericht (BMG) } \\
\text { Stand } 2017\end{array}$ & $\begin{array}{l}\text { - Verteilung der Fachgruppen der Kernteamärzte in ASV-Teams nach BL (bis zu Innere und relevante Sp), Aufstellung } \\
\text { ASV-Teams der ASV-Indikationen TBC und GIT je KV der zuständigen eLA, n und Zusammensetzung der Teams je KV } \\
\text { (auch Krankenhaus- vs. niedergelassene Ärzte), Anzahl Patienten je ASV-Team nach Indikation, Wohnort } \\
\text { - Vergütung (nur unterteilt in Krankenhaus- und Vertragsärzte) }\end{array}$ \\
\hline $\begin{array}{l}\text { Datenbank Qualitätstrans- } \\
\text { parenz (TUB) }\end{array}$ & $\begin{array}{l}\text { - Auf Abteilungsebene u. a. Angaben zur Anzahl an angestellten Ärzten, Fachärzten sowie den Fallzahlen (DRGs und } \\
\text { OPS). Die Datenbank basiert auf den Daten der strukturierten Qualitätsberichte und umfasst alle deutschen Kranken- } \\
\text { häuser ab } 2006\end{array}$ \\
\hline DRG Statistik & - z. B. Sonderauswertungen zu Verlegungen, ggf. bis 2019 \\
\hline InEF-Daten nach §23 KHG & - z.B. Detailauswertung für bestimmte Diagnosen/Prozeduren \\
\hline $\begin{array}{l}\text { KBV Honorarberichte, ggf. } \\
\text { weitere Daten }\end{array}$ & $\begin{array}{l}\text { - Übersicht Anzahl Ärzte, Fälle, Leistungsbedarf, Auszahlungsquote etc. (gesamt und je Arzt) nach Bundesländern } \\
\text { sowie nach Facharztgruppe inkl. SP von 2009/1 bis 2015/4 } \\
\text { - Übersicht TOP Behandlungsanlässe und TOP GOP nach Facharztgruppe, inkl. SP nur vereinzelt in } 2012 \text { und } 2015\end{array}$ \\
\hline MVZ-Survey (KBV) & $\begin{array}{l}\text { - Gesamtkennzahlen, Räumliche Ansiedlung, Trägerschaft, Regionale Verteilung, Verteilung der Fachgruppen (wenig } \\
\text { Detailtiefe) ab } 2006 \text { (2004-2006 nur grobe Gesamtentwicklung) } \\
\text { - nicht repräsentativ }\end{array}$ \\
\hline NRW-Gutachten & $\begin{array}{l}\text { - Daten gemäß § } 21 \text { Abs. } 3 \text { Nr. } 3 \text { KHEntgG für NRW, } 2017 \text { - ausgewertet für Leistungsbereiche und Leistungsgruppen } \\
\text { (d. h. DRGs nach inhaltlicher Gruppierung) }\end{array}$ \\
\hline $\begin{array}{l}\text { Zi Praxispanel (ggf. weitere } \\
\text { Daten) }\end{array}$ & $\begin{array}{l}\text { - Übersicht Anzahl Praxen, Patientenaufkommen und Versichertenstatus nach Praxen } \\
\text { - Nach Facharztgruppe und SP von } 2018 \text { bis } 2018 \\
\text { - nicht repräsentativ }\end{array}$ \\
\hline
\end{tabular}


Es folgt eine Übersicht der Anzahl von Ärzten und Praxen (Einzelpraxen, Berufsausübungsgemeinschaften, MVZ), das Aufkommen von Fällen und Behandlungstagen sowie von Leistungsbedarf und Auszahlungsquote, die nach Schwerpunkten der Inneren Medizin gegliedert sind und im Zeitverlauf dargestellt werden. Zudem sollen die jeweils häufigsten Behandlungsanlässe (und Gebührenordnungspositionen) der einzelnen Schwerpunkte beschrieben und in Beziehung zu denen aus dem stationären Bereich gesetzt werden.

Unter "Schnittstelle ambulant - stationär" fallen u. a. Medizinische Versorgungszentren (insbesondere die in Krankenhausträgerschaft), ambulante Versorgung durch ermächtigte Ärzte (§116 SGB V), die ambulante spezialfachärztliche Versorgung ( $\$ 116 \mathrm{~b}$ SGBV), die Hochschulambulanzen ( $\$ 117$ SGBV), stationäre Versorgung durch Belegärzte ( $§ 121$ SGB V) bzw. in Praxiskliniken ( $\$ 122$ SGB V), vor- und nachstationäre Behandlung (§115a SGB V) und ggf. ambulante Operationen ( $\$ 115 b$ SGB V). Diese sollen ebenfalls hinsichtlich ihrer personellen und organisatorischen Strukturen sowie des Fallaufkommens analysiert werden, wenngleich auch die Datenlage hier eingeschränkter ist.

Für einen internationalen Vergleich werden einige der zuvor ermittelten Kennzahlen der Inneren Medizin einem internationalen Vergleich unterzogen. Dafür werden exemplarisch Daten aus frei zugänglichen Datenbanken, z. B. der OECD, aus Ländern herangezogen, die eine ähnliche Bevölkerungsstruktur sowie eine sektorenübergreifende und umfassende Versorgungsplanung aufweisen. Dazu gehören z. B. Dänemark, Österreich und die Schweiz.

\section{Perspektiven}

Perspektive dieses Projekts ist die Erhebung und Analyse eines Datensatzes, der eine bedarfsorientierte Entwicklung der Schwerpunkte in der Inneren Medizin aufzeigt und der dann regelmäßig aktualisiert werden kann (Monitor). Dies könnte Grundlage für eine berufs- und gesundheitspolitische-Diskussion sein, um am Ende am Beispiel der Inneren Medizin medizinisch und volkswirtschaftlich sinnvolle Versorgungskonzepte zu etablieren und weiterzuentwickeln.

Autoren: Dirk Müller-Wieland, Elke Berger, Philipp Hengel, Reinhard Busse, Sebastian Schellong, Georg Ertl, Nobert Suttorp

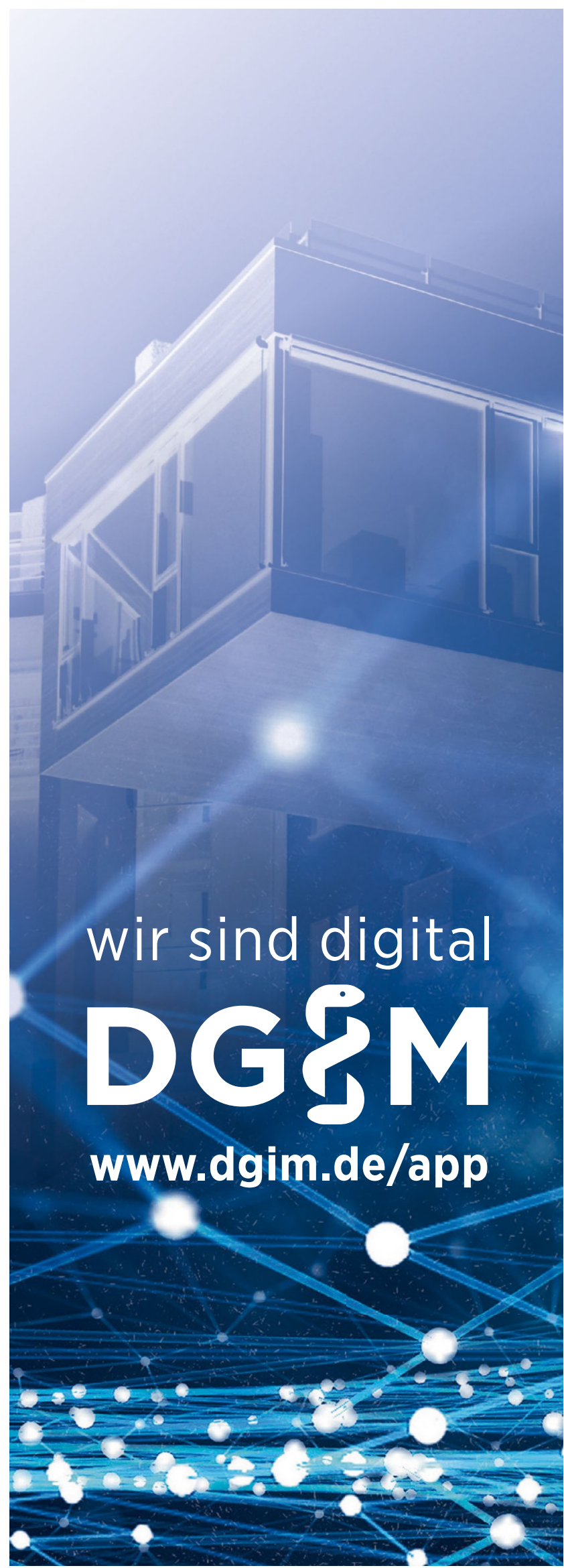




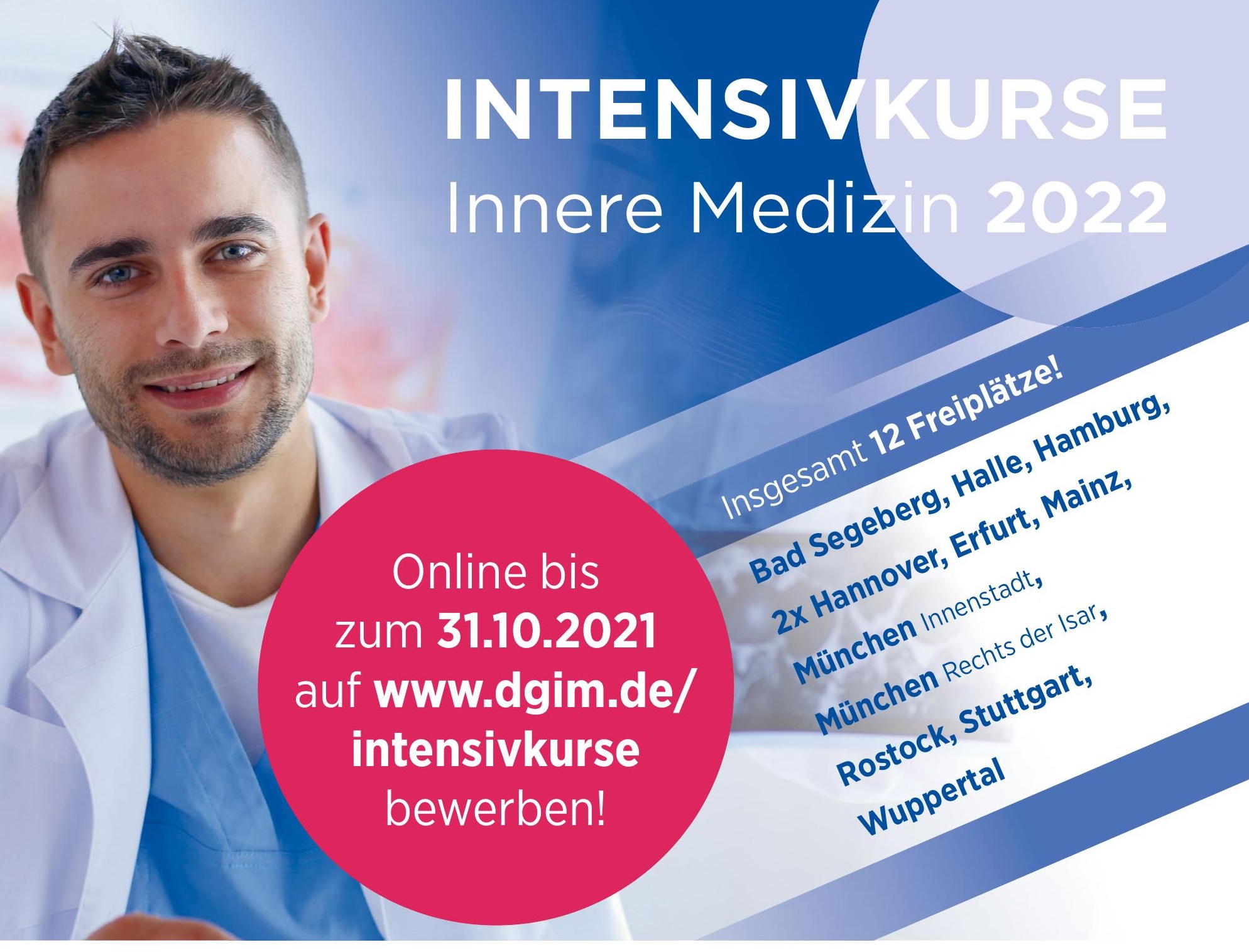

Die Deutsche Gesellschaft für Innere Medizin e.V. (DGIM) fördert Ärzte in der Vorbereitung auf die Facharztprüfung und bezuschusst die Reisekosten mit bis zu 250 Euro für das Jahr 2022. Zusätzlich stellen die einzelnen Veranstalter der Intensivkurse Innere Medizin jeweils einen Freiplatz zur Verfügung.

\section{Teilnahmebedingungen:}

$\checkmark$ Angabe des Weiterbildungsjahres (Ärztinnen und Ärzte ab dem 4. Weiterbildungsjahr)

$\checkmark$ Lebenslauf (unter Angabe des Berufsziels, z.B. Forschung, Klinik, Praxis)

$\checkmark$ Mitgliedschaft in der DGIM

$\checkmark$ Bekanntgabe bisher verfasster wissenschaftlicher Publikationen

$\checkmark$ Eine Mitarbeit in der AG JUNGE DGIM wird empfohlen

Hinweis: Nur vollständige Bewerbungen können berücksichtigt werden. Die Vergabe der Freiplätze obliegt dem Generalsekretär. Der Rechtsweg ist ausgeschlossen. 


\section{Arbeitsgemeinschaft Transition - ein Update}

Unter Transition versteht man den geplanten Übergang von Kindern oder jungen Erwachsenen mit chronischen Erkrankungen von einer kindzentrierten hin zu einer erwachsenenorientierten Gesundheitsversorgung. 2012 wurde die Arbeitsgemeinschaft (AG) Transition gegründet und besteht heute aus Vertreterinnen und Vertretern der Deutschen Gesellschaft für Kinder- und Jugendmedizin (DGKJ), der Deutschen Gesellschaft für Innere Medizin (DGIM), der Deutschen Gesellschaft für Neurologie, dem Berufsverband der Kinder- und Jugendärzte (BVKJ), der Deutschen Gesellschaft für Sozialpädiatrie und Jugendmedizin (DGSPJ), der Gesellschaft für Transitionsmedizin und dem „Berliner TransitionsProgramm“ (BTP). Die Mitglieder der AG Transition treffen sich aktuell zweimal jährlich, um sich über die verschiedenen Aktivitäten in dem Bereich Transition auszutauschen und das Feld aktiv weiter zu entwickeln.

Bereits vor zehn Jahren wurde das BTP als eine Modellstruktur für die Transition identifiziert (www.btp-ev.de). Das BTP optimiert Versorgungsstrukturen und -abläufe und ist daher kein indikationsspezifisches Modell. Die einzelnen Elemente des Programms sind mittlerweile in einem Buch zusammengefasst [1]. Während das Programm zunächst nur auf Berlin und wenige Erkrankungen beschränkt war, ist die Anzahl der Erkrankungen zwischenzeitlich deutlich gestiegen. Deshalb wurden verschiedene Modelle entwickelt, durch die akkreditierte Transitionsstellen auch in anderen Regionen Deutschlands entstanden sind und zumindest dort eine strukturierte Transition durchgeführt werden kann. Während die Kernelemente, die für die Transition benötigt werden, durch das BTP gut definiert sind und in der Akkreditierung festgehalten werden, basiert die Abrechnung dieser Leistungen weiterhin auf direkten Verhandlungen mit den Krankenkassen.

Es existiert darüber hinaus eine Reihe von zusätzlichen Programmen, die insbesondere auf Schulung von Patientinnen und Patienten abzielen und alle vom Innovationsfonds des Gemeinsamen Bundesausschusses (GBA) gefördert wurden. Hierzu gehört das TransFITProgramm, welches als Ziel hat, ein gesundheitsspezifisches Wissen aufzubauen und damit die Selbstständigkeit der Jugendlichen im Umgang mit ihrer chronischen Erkrankung zu fördern und damit den Prozess der Transition zu unterstützen (https://www.innovationtransfit.de). Im Projekt TRANSLATE-NAMSE (https://translate-namse.charite.de) zielte das Modul zur Transition darauf ab, die Gesundheitskompetenz der jugendlichen Patientinnen und Patienten mit seltenen Erkrankungen zu verbessern, indem multidisziplinäre Schulungsmaßnahmen basierend auf einer strukturierten Bedarfserfassung angeboten wurden [2]. Ein weiteres Projekt, das ebenfalls der Patient*innenschulung dient, ist AOKTrio (https://www.aok-trio.de). Hier wurde ein Onlinecoach entwickelt, der den Jugendlichen helfen soll, ihre Gesundheitskompetenz zu verbessern und mehr Eigenverantwortung für ihre Gesundheit zu übernehmen.

Diese zahlreichen Aktivitäten und ihre Bedeutung für die alltägliche klinische Praxis spiegeln sich exzellent in der gerade erschienenen S3-Leitlinie „Transition von der Pädiatrie in die Erwachsenenmedizin" der Gesellschaft für Transitionsmedizin in Zusammenarbeit mit zahlreichen Fachgesellschaften aus der Erwachsenen- sowie der
Kinder- und Jugendmedizin wider [3]. Ziel dieser Leitlinie ist es, den Prozess der Transition von der pädiatrischen Versorgung in die Versorgung der Erwachsenenmedizin für chronisch kranke Jugendliche und junge Erwachsene fachübergreifend zu strukturieren. „Damit“, so die Leitlinie, „,soll eine kontinuierliche spezialisierte medizinische Betreuung über die vulnerable Lebensphase der Adoleszenz bis in das Erwachsenenalter hinein sichergestellt werden". Anknüpfend an die Bemerkungen zu dem BTP soll auch die S3-Leitlinie dazu führen, dass Transitionsleistungen zur Regelversorgung gehören und damit auch gesichert von den Kostenträgern finanziert werden sollten.

Die dargestellten Aktivitäten zeigen auf, dass das Thema Transition kein Nischenthema mehr ist, sondern in diesen nun fast zehn Jahren Gremienarbeit der AG Transition an Sichtbarkeit gewonnen hat. Der Austausch über aktuelle Aktivitäten und das damit entstandene multidisziplinäre Netzwerk aus Kinder-, Jugend- und Erwachsenenmedizinern wird auch die kommenden Projekte begleiten und unterstützen.

\section{Autoren: Britta Siegmund, Hans-Georg Koch für die AG Transition}

\section{Literatur}

1. Findorff J, Müther S, Moers A, Nolting H-D, Burger W (2016) Das Berliner TransitionsProgramm, Sektorenübergreifendes Strukturprogramm zur Transition in die Erwachsenen Medizin. https://doi.org/10.1515/9783110432619

2. Grasemann C, Matar N, Bauer J et al (2020) Ein strukturierter Versorgungspfad von der Pädiatrie in die Erwachsenenmedizin für Jugendliche und junge Erwachsene mit einer seltenen Erkrankung. Monatsschr Kinderheilkd. https:// doi.org/10.1007/s00112-020-00929-5

3. Gesellschaft für Transitionsmedizin. S3-Leitlinie: Transition von der Pädiatrie in die Erwachsenenmedizin. Version 1.1 vom 22.04.2021. Verfügbar: https://www. awmf.org/leitlinien/detail/ll/186-001.html. Zugegriffen: 11. Jul. 2021
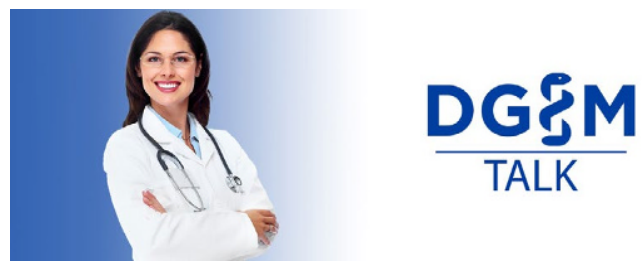

\section{DGIM Talk: Lehren aus Corona}

\section{Sendungsinhalte:}

- Neue Viren: Wie kam es zur Corona-Pandemie und was haben wir in Zukunft zu erwarten?

- Lehren aus SARS-CoV-2 und für andere respiratorische Viren: Effektiver Schutz und Therapie

- Immunsuppression und Corona: Erfahrungen für die Erkrankung und die Impfung

- Entwicklung von neuen Impfoffen: Lehren aus der CoronaPandemie

Sendetermin: Montag, 06.09.2021, 18.00 bis $19.30 \mathrm{Uhr}$

Die Online-Veranstaltung ist zertifiziert durch die LÄK Hessen mit zwei CME-Punkten.

Anmeldung unter www.streamed-up.com/anmelden. 


\section{DG?ִM e.Akademie}

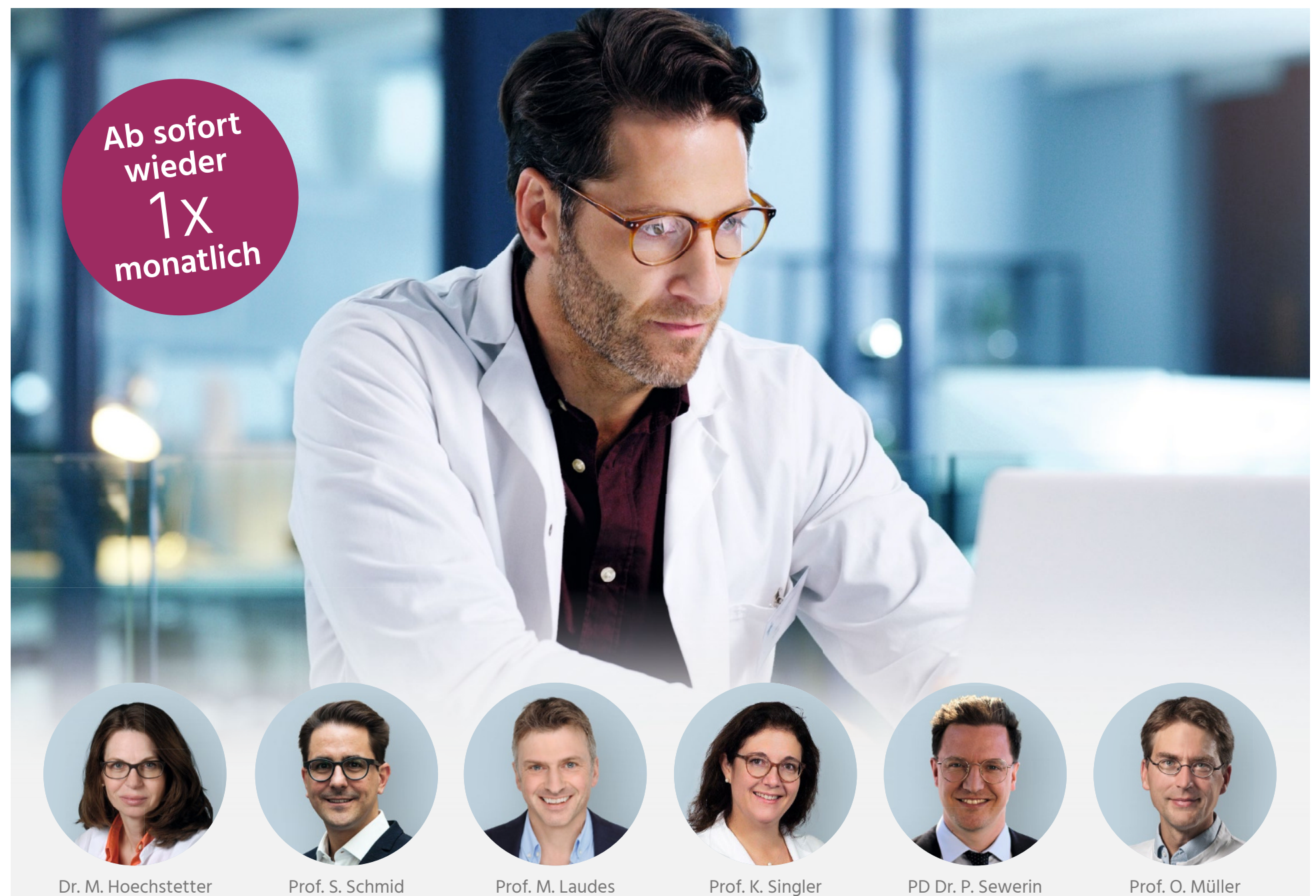

\section{Live-Webinare Innere Medizin}

Kostenfrei für DGIM-Mitglieder*

Die Webinare der DGIM e.Akademie machen zertifizierte Fortbildung so interaktiv und spannend wie nie. Erleben Sie Top-Medizinerinnen und Top-Mediziner zu den wichtigsten Themen der Inneren Medizin live oder rufen Sie unsere Webinare zu einem beliebigen Zeitpunkt einfach als Video ab.

$(8$ Aktuelle Themen: Delir des älteren Menschen, Management der frühen rheumatoiden Arthritis, Antikoagulation nach venöser Thromboembolie, Zielgerichtete Ernährungsmedizin, Leitliniengerechte Therapie des Typ 2 Diabetes, Chronische lymphatische Leukämie

() Krankheitsbilder leitliniengerecht erschließen, interaktiv Patientenfälle im Kollegenkreis lösen, im Chat Fragen stellen und mitdiskutieren

DGIM-eAkademie.de/webinare

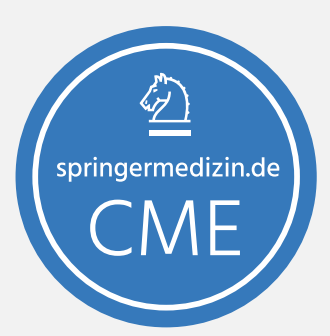

Keine Fortbildung verpassen: Jetzt den Newsletter der DGIM e.Akademie abonnieren! 


\section{Freikarten für Update- Seminare}

Die DGIM verlost unter ihren Mitgliedern 65 Freikarten für die beliebten Update-Seminare für das Jahr 2022 sowie zusätzlich 20 eBooks „Innere Medizin 2021/2022“. Zu gewinnen gibt es 20 Freikarten auf das Internisten Update 2022 in Wiesbaden und Köln. 45 Freikarten werden auf die restlichen internistischen Update-Seminare verteilt. Die DGIM versucht das Wunsch-Update-Seminar der Gewinner zu berücksichtigen, ansonsten gilt das Zufallsprinzip. Es besteht keine Garantie für die Teilnahme am gewünschten

Fachupdate-Seminar. Wer Interesse am Gewinnspiel hat, sollte sich noch bis zum 31. Oktober 2021 unter www.dgim.de/gewinnspiel anmelden. Der Rechtsweg ist ausgeschlossen. Alle Gewinner werden bis 15. Dezember 2021 schriftlich von med update benachrichtigt.

Weitere Infos unter www.med-update.com

DGIM begrüßt ihr 28.000. Mitglied

\section{Größte medizinisch-wissen- schaftliche Fachgesellschaft für Internisten in Europa wächst weiter}

Mit dem Beitritt von Verena Lüke und Melek Kücük hat die Fachgesellschaft Anfang Juli die Marke von 28.000 Mitgliedern überschritten. Die beiden Internistinnen (43 und 41 Jahre) haben kürzlich ihre Hausarztpraxis in Bochum eröffnet.

Die Übernahme der Hausarztpraxis in Bochum war für Verena Lüke und Melek Kücük der Anlass, in die DGIM einzutreten. Da sei das Angebot der DGIM sehr hilfreich, um schnell aktuelle und geprüfte Informationen rund um die Arbeit zu erhalten, so die neu-

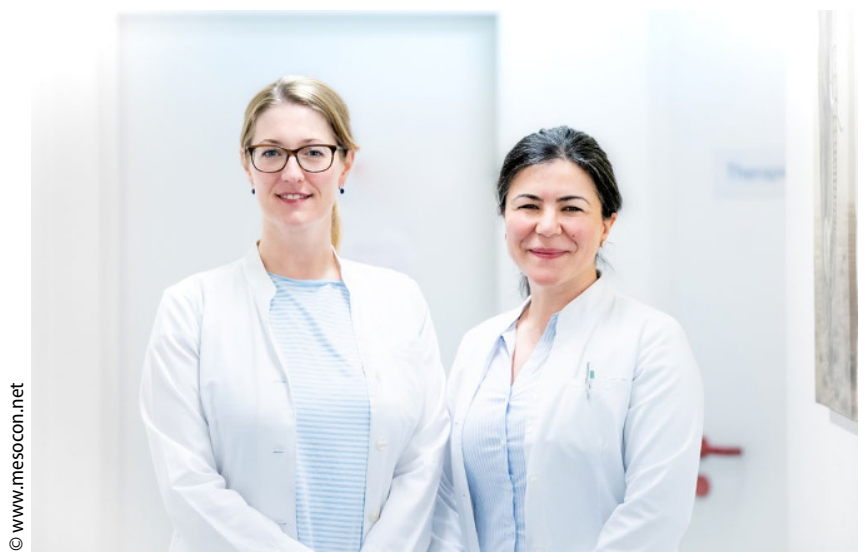

$\Delta$ Mit dem Beitritt von Verena Lüke und Melek Kücük hat die DGIM Anfang Juli die Marke von 28.000 Mitgliedern überschritten en Mitglieder. Im nächsten Schritt möchten die Praxispartnerinnen auch der AG Hausärztliche Internisten der DGIM beitreten. Diese wurde vor drei Jahren ins Leben gerufen. Ziel der AG ist es, die Interessen der rund 16.000 in Deutschland hausärztlich tätigen Internistinnen und Internisten innerhalb der DGIM zu vertreten und innen eine Plattform für Diskussion und Austausch bereitzustellen.

„Wir bieten unseren Mitgliedern den Zugang zu einer starken und aktiven Gemeinschaft", erklärt Maximilian Broglie, Geschäftsführer der DGIM. „Dabei haben wir Medizinerinnen und Mediziner jeder Karrierestufe im Blick. Wir möchten sie für die Innere Medizin begeistern und auf ihrem Weg in Klinik, Praxis und Forschung bestmöglich unterstützen."

\section{Aktuelle Ausschreibungen der DGIM}

Theodor-Frerichs-Preis

Den Preis verleiht die DGIM für die beste vorgelegte möglichst klinisch-experimentelle Arbeit auf dem Gebiet der Inneren Medizin. Er ist mit 30.000 Euro dotiert. Bewerbungsfrist: 15. Oktober 2021

12 Stipendien für die Intensivkurse Innere Medizin Die DGIM fördert Ärztinnen und Ärzte in der Vorbereitung auf die Facharztprüfung Innere Medizin und bezuschusst die Reisekosten mit bis zu 250 Euro für das Jahr 2022. Bewerbungsfrist: 31. Oktober 2021.

\section{Präventionspreis}

Den Preis verleiht die DGIM gemeinsam mit der Deutschen Stiftung Innere Medizin für die beste vorgelegte Arbeit auf dem Gebiet der Primär- und Sekundärprävention. Er ist mit 10.000 Euro dotiert. Bewerbungsfrist: 15. November 2021.

\section{Peter-Müller-Kreativ-Preis Medizin}

Mit dem Peter-Müller-Kreativ-Preis Medizin würdigt die DGIM kreative und innovativ denkende Personen, Organisationen oder Firmen, insbesondere im Bereich der digitalen Medizin. Der Preis wird von der Firma custo med $\mathrm{GmbH}$ gestiftet. Es können auch mögliche Preisträger vorgeschlagen werden. Bewerbungsfrist: 15. November 2021.

\section{Leopold-Lichtwitz-Medaille}

Zu Ehren herausragender Ärzte und Forscher verleiht die DGIM die Leopold-Lichtwitz-Medaille. Vorschlagsberechtigt ist jedes ordentliche Mitglied der DGIM. Einsendeschluss: 15. Dezember 2021.

\section{Peter Scriba-Promotionsstipendien}

Mit den Stipendien fördert die DGIM den wissenschaftlichen Nachwuchs bei anspruchsvollen, arbeitsintensiven und wissenschaftlich hochwertigen klinisch orientierten Promotionsarbeiten auf dem Gebiet der Inneren Medizin unter Berücksichtigung aller Schwerpunkte. Die Fachgesellschaft vergibt bis zu fünf Stipendien in einer Höhe von jeweils bis zu 10.000 Euro. Bewerbungsfrist: 28. Februar 2022.

2 Stipendien für das Clinician Scientist Programm Mit diesem Stipendium fördert die DGIM die Freistellung eines Stipendiaten von der ärztlichen Tätigkeit und ermöglicht damit Freiräume für wissenschaftliches Arbeiten. Der Förderumfang beträgt bis zu 100.000 Euro. Bewerbungsfrist: 28. Februar 2022

2 Stipendien für das Advanced Clinician Scientist Programm Die DGIM möchte durch die Vergabe eines Advanced Clinician Scientist Stipendiums im Jahr 2022 durch eine Freistellung von der klinischen Tätigkeit beste Voraussetzungen für wissenschaftliches Arbeiten schaffen. Die maximale Gesamtförderung durch die DGIM beträgt für die Förderdauer von längstens vier Jahren 120.000 Euro. Bewerbungsfrist: 28. Februar 2022

Weitere Informationen unter www.dgim.de/nachwuchsfoerderung und www.dgim.de/Preise 


\section{Kongress Südwestdeutsche Gesellschaft für Innere Medizin}

\author{
08.-09.10.2021 in Heidelberg
}

Liebe Kolleginnen und Kollegen,

zum 58. Kongress der Südwestdeutschen Gesellschaft für Innere Medizin möchten wir Sie herzlich für den 08. und 09.10.2021 nach Heidelberg einladen. Nachdem unser letzter Kongress auf Grund der COVID-19 Pandemie verschoben werden musste, blicken wir für 2021 nach neuesten Entwicklungen optimistisch nach vorn. Durch starke nationale und internationale medizinische und biologische Forschungsanstrengungen konnten in den letzten Monaten mehrere Studien zügig realisiert und unter anderem erste SARS-CoV-2 Impfstoffe produziert werden. Da der SWGIM-Kongress neben Vorträgen vor allem von regen Diskussionen, persönlichem Austausch, Postersessions und praktischen Kursen lebt, freuen wir uns über diese vielversprechenden Ansätze und planen unseren 58. Kongress als Hybridveranstaltung. Unser Jahreskongress wird durch ein vielseitiges Programm mit praxisrelevanten Themen definiert. Es besteht die Möglichkeit, sich im Querschnitt aktuellster internistischer Bereiche fortzubilden. Wir freuen uns auf den gemeinsamen Kongress mit hochkarätigen neuen Forschungsergebnissen und regem Austausch sowohl zwischen jungen Kollegen als auch Mitgliedern mit langjähriger Erfahrung. Wir verbleiben bis dahin mit besten Grüßen Dirk Jäger und Martin Zeier

\section{Tagungspräsidenten}

Prof. Dr. med. Dirk Jäger

NCT Managing and Medical Director

National Center for Tumor Diseases (NCT) Heidelberg

Head of Medical Oncology

Heidelberg University Hospital (UKHD)

Head of Clinical Cooperation Unit „Applied Tumor-Immunity“

German Cancer Research Center (DKFZ)

Prof. Dr. med. Martin Zeier

Ärztlicher Direktor

Nierenzentrum Heidelberg

\section{Organisation}

Aey Congresse GmbH Seumestr. 810245 Berlin Telefon: +49302900 6594 Telefax: +49 3029006595 E-Mail: swgim@aey-congresse.de

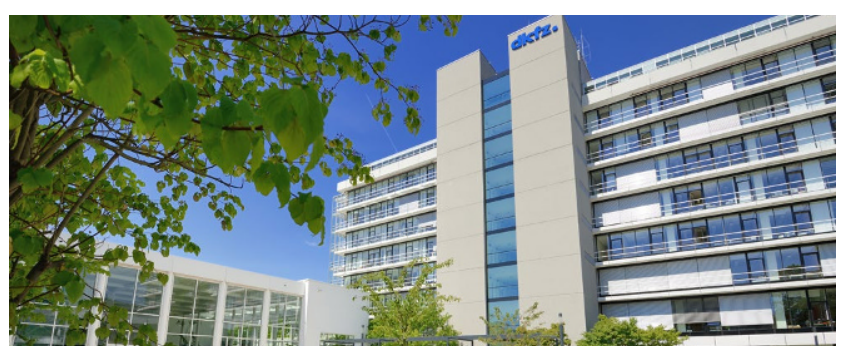

Preise

Ludolf-Krehl-Preis Abteilung A (3000 Euro) für Doktoranden, die an einer deutschen Universität in den letzten zwei Jahren auf dem Gebiet der Inneren Medizin mit dem Prädikat „magna cum laude“ oder „summa cum laude" promoviert haben.

Ludolf-Krehl-Preis Abteilung B (3000 Euro) für die besten Arbeiten von Assistenz- und Oberärzten aus südwestdeutschen Krankenhäusern (ausgenommen Universitätskliniken), die in den letzten zwei Jahren in einer wissenschaftlichen Zeitschrift veröffentlicht worden sind.

Die besten Poster werden mit jeweils 500 Euro ausgezeichnet.

\section{Programm}

I.

COVID

Patho-anatomische Befunde bei COVID-19-Infektion

- klinische Implikationen P. Schirmacher; Heidelberg Impfung und Impfstoffentwicklung R. Bartenschläger; Heidelberg Therapieoptionen bei kritisch COVIDKranken (antiviral, immunmodulatorisch, invasiv) U. Merle; Heidelberg

Beatmungsstrategien - die intensivmedizinische Sicht M. Fiedler, Heidelberg

II. Onkologie

Zellbasierte Therapien beim Multiplen Myelom H. Goldschmidt, Heidelberg Immunonkologie - neue Strategien St. Zschäbitz, Heidelberg

Was kann die personalisierte Medizin in der Onkologie leisten? Fröhling, Heidelberg

Die Rolle von Sport und Bewegung in der Onkologie R. Kühl, Heidelberg

III. Gastroenterologie

NASH- schlimmer als Alkohol? Böttler, Freiburg

Hepatitis E D. Bettinger, Freiburg

Neue Entwicklungen in der Endoskopie

L. Goßner, Karlsruhe IV.

Kardiologie und Angiologie Update Herzinsuffizienz N. Frey, Heidelberg

Thrombektomie und Stentversorgung des venösen Systems Ch. Erbel, Heidelberg Interventionelle Therapie der Trikuspidal-Klappe R. Bekeredjian, Stuttgart

SGLT-2 Inhibitoren - welchen Stellenwert erreichen sie in der kardiovaskulären Medizin? J. Galle, Lüdenscheid

V. Nephrologie und Rheumatologie

Neue Immunsuppressiva bei Arthritis und Spondylarthritis - Schultz, Baden-Baden

Aktuelle Studien und neue therapeutische Ansätze bei Vaskulitiden R. Bergner, Ludwigshafen

Aktueller Stand der Behandlung von Glomerulonephritiden J. Floege, Aachen

VI. Pulmonologie Update COPD F. Herth, Heidelberg Update Lungenkrebs A. Tufman, München 


\section{ENDOKRINO UPDATE 2021}

\section{Wissen kompakt und topaktuell!}

\section{Endokrinologie-Update-Seminar}

Das Endokrino Update bietet Ihnen in nur zwei Tagen einen umfassenden Überblick über die wichtigsten Forschungsergebnisse des zurückliegenden Jahres im Fachbereich der Endokrinologie. In 14 Teilseminaren stellt Ihnen das

Team aus ausgewählten Referierenden die wichtigsten Studien des vorangegangenen Jahres vor, fasst diese kompakt zusammen und analysiert sie kritisch für Ihre tägliche $\mathrm{Ar}$ beit. Mit den in die Vorträge integrierten Diskussionspau-

\section{Programm}

Hypophyse

Martin Reincke, München

Nebenniere

Martin Fassnacht, Würzburg

Calcium- und Knochenstoffwechse

Elena Tsourdi, Dresden

Hot Topic: Andrologie

Sabine Kliesch, Münster

Hot Topic: Endokrinologie im Alter

Joachim Feldkamp, Bielefeld

Diabetes I: Prävention und Therapie

Jens Aberle, Hamburg

Schilddrüse I: Funktion, Hormone, Autoimmunerkrankung

Jörg Bojunga, Frankfurt a. M.

Endokrine Onkologie

Harald Lahner, Essen

Hot Topic: Pädiatrische Endokrinologie und päd.

Diabetologie inkl. Transition · Joachim Wölfle

Lipidologie

Anja Vogt, München

Adipositas

Matthias Blüher, Leipzig

Diabetes II: Grundlagen und neue Entwicklungen

Sebastian Schmid, Lübeck

Gynäkologische Endokrinologie

Michael Ludwig, Hamburg

Schilddrüse II: Knoten, Struma, Krebs

Dagmar Führer, Essen sen und den Speakers' Corners, in denen die Referierenden für Fragen im kleinen Kreis zur Verfügung stehen, bietet die Veranstaltung zahlreiche Gelegenheiten für den Austausch mit den Expertinnen und Experten und dem Auditorium. Neu im Programm sind die von den Teilnehmenden des Vorjahres gewünschten Hot Topics „Pädiatrische Endokrinologie und päd. Diabetologie inkl. Transition“, „Andrologie“ und „Endokrinologie im Alter“.

Zur Vertiefung der Seminarinhalte und als praktisches Nachschlagewerk erhalten alle Teilnehmenden das Handbuch UPDATE ENDOKRINOLOGIE 2021 als Druckausgabe oder im eBook-Format mit den Manuskripten aller Referierenden - außerdem stehen sämtliche Vorträge als eCharts im Nachgang zum Download bereit. Neben der Präsenzveranstaltung steht Ihnen das Endokrino Update auch als interaktiver Livestream zur Verfügung! Den Teilnehmenden des Endokrino Updates winkt noch ein weiteres Plus: Auf der Videoplattform "streamedup!« stehen Ihnen die Aufzeichnungen aller Vorträge des Updates kostenfrei zur Verfügung.

Schirmherren des Endokrino Updates sind die DGIM und die DGFF (Lipid-Liga). Unternehmen aus der Industrie unterstützen das Seminar, ohne dabei Einfluss auf die Inhalte zu nehmen.

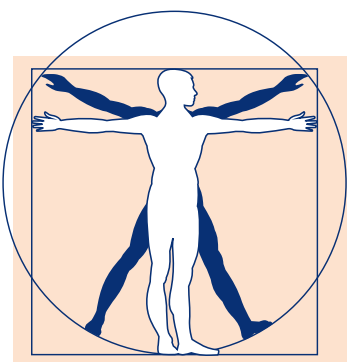

2. Endokrinologie-UpdateSeminar

24. und 25. September 2021 Berlin

24. und 25. September 2021, Livestream

Wissenschaftliche Leitung Matthias Blüher, Leipzig Jörg Bojunga, Frankfurt

Martin Fassnacht, Würzburg

Dagmar Führer, Essen

Alle Veranstaltungen parallel als Livestream

UPDATE-Vorschau

Hepato Update

11.-12.06.2021, Mainz

hepato-update.com

Praxis Update

11.-12.06.2021, Hamburg

18.-19.06.2021, Mainz

18.-19.06.2021, München

praxis-update.com

Intensiv Update

24.-25.09.2021, Köln

intensiv-update.com

Internisten Update

05.-06.11.2021, Hamburg

19.-20.11.2021, Köln

19.-20.11.2021, München

26.-27.11.2021, Wiesbaden

26.-27.11.2021, Berlin

internisten-update.com

Pneumo Update

12.-13.11.2021, Mainz

19.-20.11.2021, Berlin pneumo-update.com

SCHIRMHERRSCHAFT Deutsche Gesellschaft für Innere Medizin

Überzeugt vom Update-Konzept übernimm die DGIM seit 2007 die Schirmherrschaft für alle Update-Seminare der Inneren Medizin. 


\section{Veranstaltungen unter der Schirmherrschaft der DGIM}

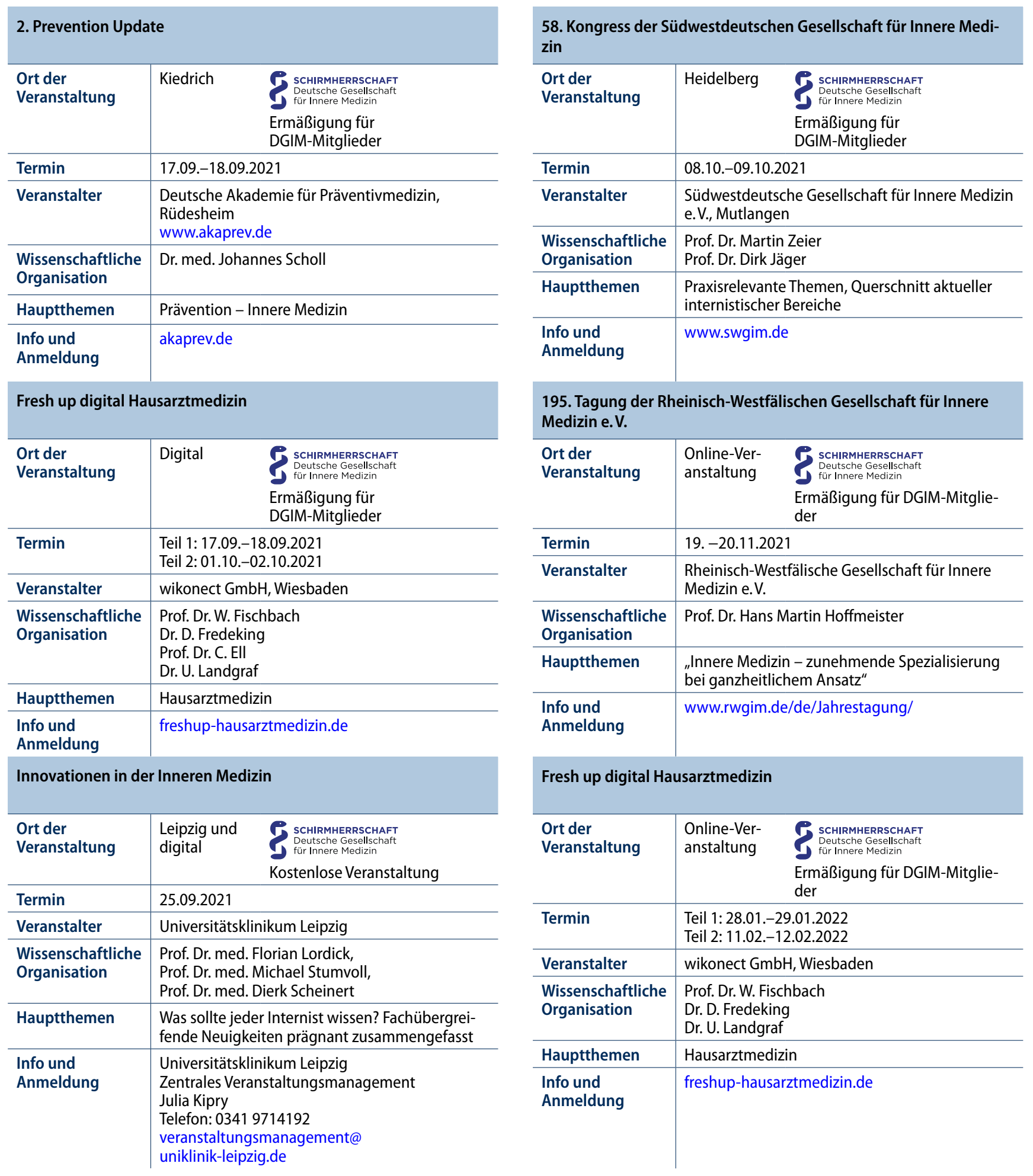




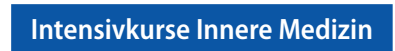

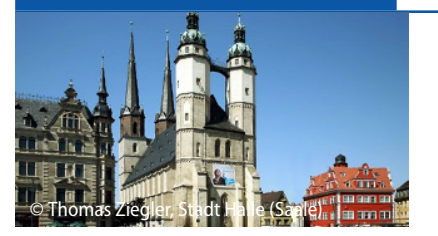

Veranstalter

Universitätsklinikum Halle

Department für Innere Medizin

Leitung

Prof. Dr. med. M. Binder

Prof. Dr. med. M. Girndt

Prof. Dr. med. P. Michl

Prof. Dr. med. D. Sedding

Anmeldung/Organisation

Universitätsklinikum Halle

Stephanie Fritsche

Department für Innere Medizin

06120 Halle/Saale

Telefon: 0345 557-4978 oder -3238

Telefax: 0345 557-904978

dim@uk-halle.de

Gebühr

Nichtmitglieder: 380,00 Euro

DGIM-Mitglieder: 350,00 Euro

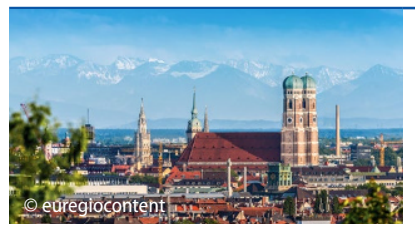

S SCHIRMHERRSCHAFT Deutsche Gesellschaft

\section{München rechts der Isar}

27.09.-01.10.2021

Veranstaltungsort

Klinikum rechts der Isar

Hörsaal A

Ismaninger Straße 22

81675 München

Gebühr

Nichtmitglieder: 440,00 Euro

DGIM-Mitglieder: 400,00 Euro

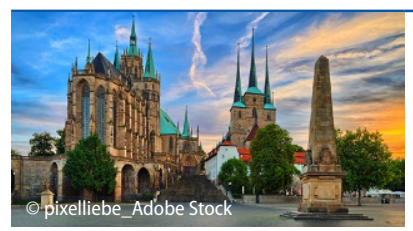

SCHIRMHERRSCHAFT
Deutsche Gesellschaft
für Innere Medizin

\section{Erfurt}

11.10.-15.10.2021

Veranstaltungsort

Helios Klinikum Erfurt

Auditorium

Nordhäuser Str. 74

99089 Erfurt

Gebühr

Nichtmitglieder: 595,00 Euro

DGIM-Mitglieder: 476,00 Euro
Veranstalter

Klinikum rechts der Isar, München

Leitung

Prof. Dr. med. R. M. Schmid

Prof. Dr. med. F. Bassermann

Prof. Dr. med. K.-L. Laugwitz

Anmeldung/Organisation

PD Dr. med. Fabian Geisler

Frau Mandy Söhner

Klinikum rechts der Isar der TUM

Klinik und Poliklinik für Innere

Medizin II

Telefon: 089 4140-2252

Telefax: 089 4140-7287

mandy.soehner@mri.tum.de

\section{Veranstalter}

Landesärztekammer

Thüringen, Jena

und Weiterbildung

Leitung

Prof. Dr. med. J.-G. Scharf

Anmeldung/Organisation

HELIOS Klinikum Erfurt GmbH

2. Medizinische Klinik

Frau Iris Effenberger

Nordhäuser Str. 74

99089 Erfurt

Telefon: 03617812471

Fax: 03617812472

iris.effenberger@

helios-gesundheit.de

www.helios-gesundheit.de
Akademie für ärztliche Fort-

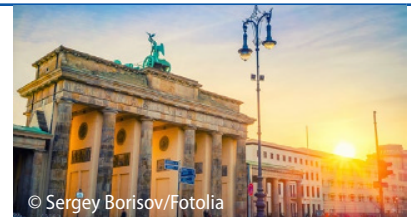

Veranstalter

Medizinische Klinik I

Universitätsklinikum Lübeck

Campus Lübeck

Ratzeburger Allee 160

23562 Lübeck

Leitung

Prof. Dr. G. Gerken

Prof. Dr. G. Hintze

Prof. Dr. H. Lehnert

Prof. Dr. N. Suttorp

Anmeldung/Organisation

Frau D. Henze

Tel.: +49 1704904675

Fax: +4939162029414

intensivkurs-berlin@arcor.de

www.persberg.de

Nichtmitglieder: 620,00 Euro

DGIM-Mitglieder: 580,00 Euro

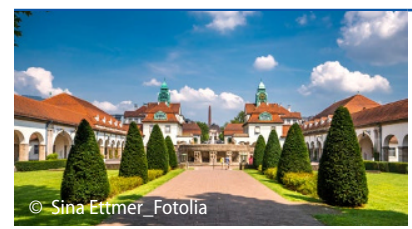

Schirmherrschaft

Deutsche Gesellscha

Bad Nauheim

15.11.-20.11.2021

Veranstaltungsort

Fortbildungszentrum der Landes-

ärztekammer Hessen

Carl-Oelemann-Weg 5

61231 Bad Nauheim

Gebühr

Nichtmitglieder: $630,00 € /$ einzel-

ner Tag je 180,00€

DGIM- und BDI-Mitglied: $567,00 € /$

einzelner Tag je 162,00€

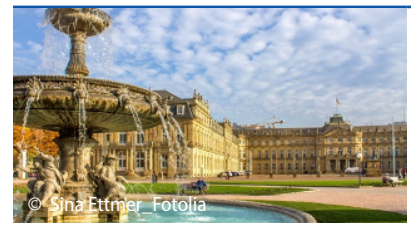

SCHIRMHERRSCHAFT
Deutsche Gesellschaft
für Innere Medizin

\section{Stuttgart}

17.01.-22.01.2022

Veranstaltungsort

ATRIUM

Kongresszentrum RBK

Auerbachstr. 110

70376 Stuttgart

Leitung

Prof. Dr. med. W. Fassbinder, Fulda

Anmeldung/Organisation

Akademie für Ärztliche Fort- und

Weiterbildung

der LÄK Hessen

Adiela Candelo-Römer

Carl-Oelemann-Weg 5

61231 Bad Nauheim

Tel.: 06032 782-227

Fax: 069 97672-67227

E-Mail: adiela.candelo-roemer@

laekh.de

http:I|www.akademie-laekh.de
Veranstalter

Prof. Dr. med. E. F. Stange, Stuttgart

Leitung

Prof. Dr. med. M.D. Alscher

Prof. Dr. med. Dr. R. Bekeredjian

Prof. Dr. med. E. F. Stange

Anmeldung/Organisation

Irmgard-Bosch-Bildungszentrum am RBK

Stephanie Bügler-Mietens

Auerbachstr. 110

70376 Stuttgart

Fax: 0711-8101-92560

rbk.de stephanie.buegler-mietens@

Gebühr

steht noch nicht fest 